\title{
Substrate Stiffness Affects Endothelial Cell Junctions and MAPK Signaling Pathway
}

\author{
Huanli Wang, Chao Zhang, Xiaomei Han, Jin Xu, Yue Zhou* \\ School of Biomedical Engineering, Shanghai Jiao Tong University, Shanghai, China \\ Email: *yzhou2009@sjtu.edu.cn
}

How to cite this paper: Wang, H.L., Zhang, C., Han, X.M., Xu, J. and Zhou, Y. (2022) Substrate Stiffness Affects Endothelial Cell Junctions and MAPK Signaling Pathway. Journal of Biosciences and Medicines, 10, 124-135.

https://doi.org/10.4236/jbm.2022.102012

Received: January 17, 2022

Accepted: February 20, 2022

Published: February 23, 2022

Copyright $\odot 2022$ by author(s) and Scientific Research Publishing Inc. This work is licensed under the Creative Commons Attribution International License (CC BY 4.0).

http://creativecommons.org/licenses/by/4.0/ (c) (i) Open Access

\begin{abstract}
Vascular homeostasis is critical for maintaining normal vascular structure and function. Aging is an irreversible trigger of vascular sclerosis, which causes structural and functional damage to blood vessels, leading to severe atherosclerosis. Endothelial cells (ECs) can respond to mechanical stimuli from the extracellular matrix, causing disruption of endothelial barrier function and activating signaling pathways to regulate cellular behavior under pathological conditions. In this paper, we investigated the effect of substrate stiffness on endothelial cell junctions, and the activation of mitogen-activated protein kinase (MAPK) signaling pathways. An in vitro stiffness model was established using polyacrylamide hydrogels of $1 \mathrm{kPa}, 20 \mathrm{kPa}$ and $100 \mathrm{kPa}$. By transcriptome analysis, we found that the cell-cell junction, cadherin binding, cytoskeleton and classical signaling pathways such as MAPK and Rho GTPase of endothelial cells were regulated by substrate stiffness. The expression of cell junction-related molecules TJP1, TJP2, JAM3 and JCAD was also found to be reduced at higher stiffness. The MAPK signaling pathway-related molecules MAP2K3, MAP2K7, MAP3K3, MAP3K6, MAPK3, MAPK7 were upregulated with increased stiffness. qRT-PCR analysis showed that the gene expression of JCAD was reduced with increased stiffness. Immunofluorescence staining of VE-cadherin indicated that the total fluorescence level of VEcadherin decreased significantly with increased stiffness, and stiffness impaired the cell-cell junction with increased punctuation and discontinuity. Western blotting analysis confirmed that the protein expression ratio of $\mathrm{p}$ p38MAPK/p38MAPK increased with stiffness. Our research suggested that substrate stiffness played an important role in regulating endothelial cell integrity and MAPK signaling pathway.
\end{abstract}

\section{Keywords}

Substrate Stiffness, Endothelial Cell, Cell Junction, MAPK Signaling Pathway 


\section{Introduction}

The vasculature forms an interface between blood circulation and different organ environments. Vascular homeostasis plays an integral role in maintaining normal tissue functions, as well as adapting to pathological challenges [1]. Previous studies have shown that vascular homeostasis is essential in development, ageing, inflammation, and metabolism. Under the influence of pathogenic factors, the internal and external environment of the vasculature is altered and vascular homeostasis is out of balance, leading to changes in vascular function and structure. Vascular dysfunction is inextricably linked to many chronical diseases, such as cancer and atherosclerosis [2] [3] [4].

As early as the 1850s, studies in anatomic pathology began to explore the consequences caused by endothelial dysfunction. They observed that lipid accumulation occurs at the site of early lesions, and local endothelial permeability was altered [5]. Subsequently, it was found that the endothelium not only acts as a selective barrier but also as a source of various autocrine factors involved in autocrine and paracrine functions [6]. The imbalance in vascular homeostasis is characterized by thickening of the vessel wall and a decrease in nitric oxide [7]. The serious consequences of imbalance in vascular homeostasis can be observed in the following diseases. Diabetes-induced endothelial dysfunction leads to decreased NO bioavailability, and endothelial dysfunction in microvascular complications is mainly manifested by decreased NO release, increased inflammatory factors, and impaired endothelial repair [8]. Atherosclerosis and coronary artery disease lead to vascular dysfunction, causing an imbalance in the ratio between vasodilator and vasoconstrictor factors, promoting thrombosis and atherosclerosis as evidenced by vasoconstriction, leukocyte adhesion, platelet activation, vascular inflammation [9] [10]. Hypertension and hypercholesterolemia are involved in vascular inflammatory processes, and under pathological conditions ROS can stimulate NF- $\kappa$ B to activate various pro-inflammatory cytokines, such as tumor necrosis factor- $\alpha$ (TNF- $\alpha$ ) and interleukin-1 (IL-1), increase the expression of cell adhesion molecules, accelerate endothelial dysfunction, and recruit monocytes into the intima, leading to chronic inflammation of the vessel wall [11] [12].

Aging is a major irreversible risk factor for vascular homeostasis and endothelial dysfunction [13]. Vascular remodeling, inflammation, calcification, and increased stiffness are specific manifestations of altered vascular homeostasis, which may become a prologue to atherosclerosis. Aging vessels often display a specific cellular morphology and molecular composition, resulting in increased vascular stiffness [14]. Akhtar et al. designed an aging model of the female sheep aorta and measured the mechanical properties of the ascending aorta in older and younger sheep using the frequency-modulated atomic force microscopy and found that the elastic modulus was approximately $113.9 \mathrm{kPa}$ in older sheep and $42.9 \mathrm{kPa}$ in younger sheep [15]. However, why alterations in vascular stiffness influence vascular homeostasis remain to be further investigated. 
It is known that vascular homeostasis is mediated by tissue-specific endothelium consisting of endothelial cells (ECs). Healthy EC monolayers have intact cell-cell junctions, and endothelium is in a dynamic process and maintaining vascular homeostasis and normal function [16]. Changes in vascular stiffness may affect EC junctions, thus affecting the integrity and function of endothelium. Therefore, in this study, we investigated the effect of vascular stiffness on EC junctions. Moreover, the possible signaling pathways associated were also explored.

\section{Materials and Methods}

\subsection{Establishment of an in Vitro Stiffness Model}

Stiffness model was used to establish an in vitro cell culture system to mimic the different stiffness condition. Acrylamide and bis-acrylamide (Sangon Biotech, China) was mixed at different ratio described in previous studies [17] [18] [19] to manufacture polyacrylamide hydrogels of different stiffnesses. The crosslinking reagents were $1 \mathrm{M}$ HEPES (Sigma, USA), 10\% APS (Sangon Biotech, China) and TEMED (Klamar, China). Sulfo-SANPAH (ProteoChem, USA) was used for UV cross-linking. The surface of the polyacrylamide hydrogel was coated with $0.1 \mathrm{mg} / \mathrm{ml}$ of rat collagen I (Corning, USA) solution at $4^{\circ} \mathrm{C}$ overnight to ensure proper cell adhesion. According to previous studies, $20 \mathrm{kPa}$ was selected as normal stiffness control. One $\mathrm{kPa}$ and $100 \mathrm{kPa}$ were selected as pathological stiffness condition [15] [18].

\subsection{Cell Culture and Seeding}

Human umbilical vein endothelial cells (HUVECs) (Sciencell, USA) were used as a representative EC in this study. The cells were maintained in Endothelial Cell Medium (ECM) provided by ScienCell (USA) in a $37^{\circ} \mathrm{C}, 5 \% \mathrm{CO}_{2}$ incubator.

Before cell seeding, the hydrogels were irradiated with UV for $30 \mathrm{~min}$ and treated with $75 \%$ alcohol for $10 \mathrm{~s}$. After washing with DPBS, the ECs were diluted to a concentration of $10 \times 102$ cells $/ \mathrm{mm}^{2}$ and uniformly added dropwise to the surface of the hydrogels and cultured in an incubator at $37^{\circ} \mathrm{C}$ with $5 \% \mathrm{CO}_{2}$.

\subsection{Transcriptome Analysis}

In this experiment, total RNA was extracted from ECs grown on softer $(1 \mathrm{kPa})$ and stiffer $(100 \mathrm{kPa})$ hydrogels and then subjected to transcriptome analysis. The genes expressed on different stiffnesses were explored by differentially expressed gene analysis and prediction analysis of transcription factors and their target genes. The transcriptome analysis was provided by the services of Oebiotech, China.

\subsection{Quantitative Real-Time PCR (qRT-PCR)}

The total RNA extraction, reverse transcription and qRT-PCR were performed using kits from Tiangen, China, followed by the instructions of the manufactur- 
er. The primers used in the experiment were purchased from BioTNT, China. The primers are as follows, human JCAD: (forward) 5'-CGG AGG CGG GGT TTT GTA AT-3', (reverse) 5'-GGG GTC ATT GAT GGC AAC A-3', human GAPDH: (forward) 5'-GGG AAG GTG AAG GTC GGA GT-3', (reverse) 5'-CAC CCT GTT GCT GTA GCC AAA TTC-3'.

\subsection{Western Blotting}

RIPA (Beyotime, China) was used as the lysis solution and protein concentration was determined using BCA Protein Assay Kit (Thermo Scientific, USA). The protein samples were denatured with loading buffer at $95^{\circ} \mathrm{C}$ for 5 minutes. The membrane was blocked using 10\% BSA at room temperature for 2 hours and incubated with primary antibodies overnight at $4^{\circ} \mathrm{C}$. The primary antibodies were p-p38MAPK, p38MAPK (both from Cell Signaling Technology, USA), and GAPDH (Proteintech, USA), respectively. Then the membrane was incubated with HRP-conjugated secondary antibody for 1.5 hours at room temperature. Protein chemiluminescence was performed using Chemiluminescent HRP Substrate (MILLIPORE, USA). The images were acquired by Biorad imaging system (USA).

\subsection{Immunofluorescence}

Cells were fixed with $4 \%$ paraformaldehyde for $15 \mathrm{~min}$ and permeabilized using $0.5 \%$ TritonX-100 at room temperature. After blocking with 5\% BSA, VE-cadherin antibody (Cell Signaling Technology, USA, 1:500) was incubated overnight at $4^{\circ} \mathrm{C}$. The fluorescence labeled secondary antibodies were incubated at room temperature for 1.5 hours. Cell nucleus were stained with DAPI (Beyotime, China, 1:5000). The images were acquired by a laser confocal microscope (Leica, TCS SP5 II, Germany) and the intensity of the signal was quantified by ImageJ V1.8.0.

\subsection{Statistical Analysis}

Unless otherwise stated, results were shown as the mean of at least three independent experiments, and error bars represent the standard error of the mean (SEM). Pairwise comparisons were performed using the Student $\mathrm{t}$-test. $\mathrm{P}$ values less than 0.05 were considered statistically significant in this experiment.

\section{Results}

\subsection{Substrate Stiffness Affected Cell-Cell Junctions Assembly and the Classical Signal Transduction Pathway MAPK}

Vascular Sclerosis is manifested by increased mitochondrial oxidative stress, increased levels of inflammatory factors, and increased vascular permeability. When vascular function is impaired, the composition and structure of the extracellular matrix is altered, leading to damage in endothelial cell-cell junctions. At the same time, signaling pathways are activated in response to external mechanical 
stimuli. We performed transcriptome analysis in ECs cultured on softer (1 kPa) and stiffer $(100 \mathrm{kPa})$ substrates, as shown by the transcriptome enrichment analysis (Figure 1). The results indicated that cell-cell junction assembly was the major biological process affected. In addition, cytoskeleton organization and focal adhesion were significantly involved as well. In view of cellular components, cell-cell junction proteins, including adherens and gap junction proteins, were all affected. As to molecular functions, cadherin binding and cell-cell junction assembly were significantly affected by substrate stiffness. Mitogen-activated protein kinase (MAPK), Rho GTPase and other classical signal transduction pathways were also regulated by substrate stiffness. In summary, the above results suggested that substrate stiffness as an important external mechanical signal has profound impact on the integrity of cell-cell junctions and the classical signaling pathway MAPK.

\subsection{Substrate Stiffness Regulated MAPK Signaling Pathway}

MAPK is a protein kinase that plays a role in cell growth, proliferation, differentiation and apoptosis. MAPK was activated by phosphorylation of its kinase MAPKK, which in turn can be activated by phosphorylation of its kinase MAPKKK. The process may involve small GTPases or other proteases, among which small GTPases are Rac, Rho, etc. Activation of the MAPK/ERK pathway in ECs at wound healing can regulate the angiogenic response of microvascular ECs. p38MAPK is activated by environmental stress or inflammatory cytokines, which in turn regulate cell behavior and function. Transcriptome analysis of ECs

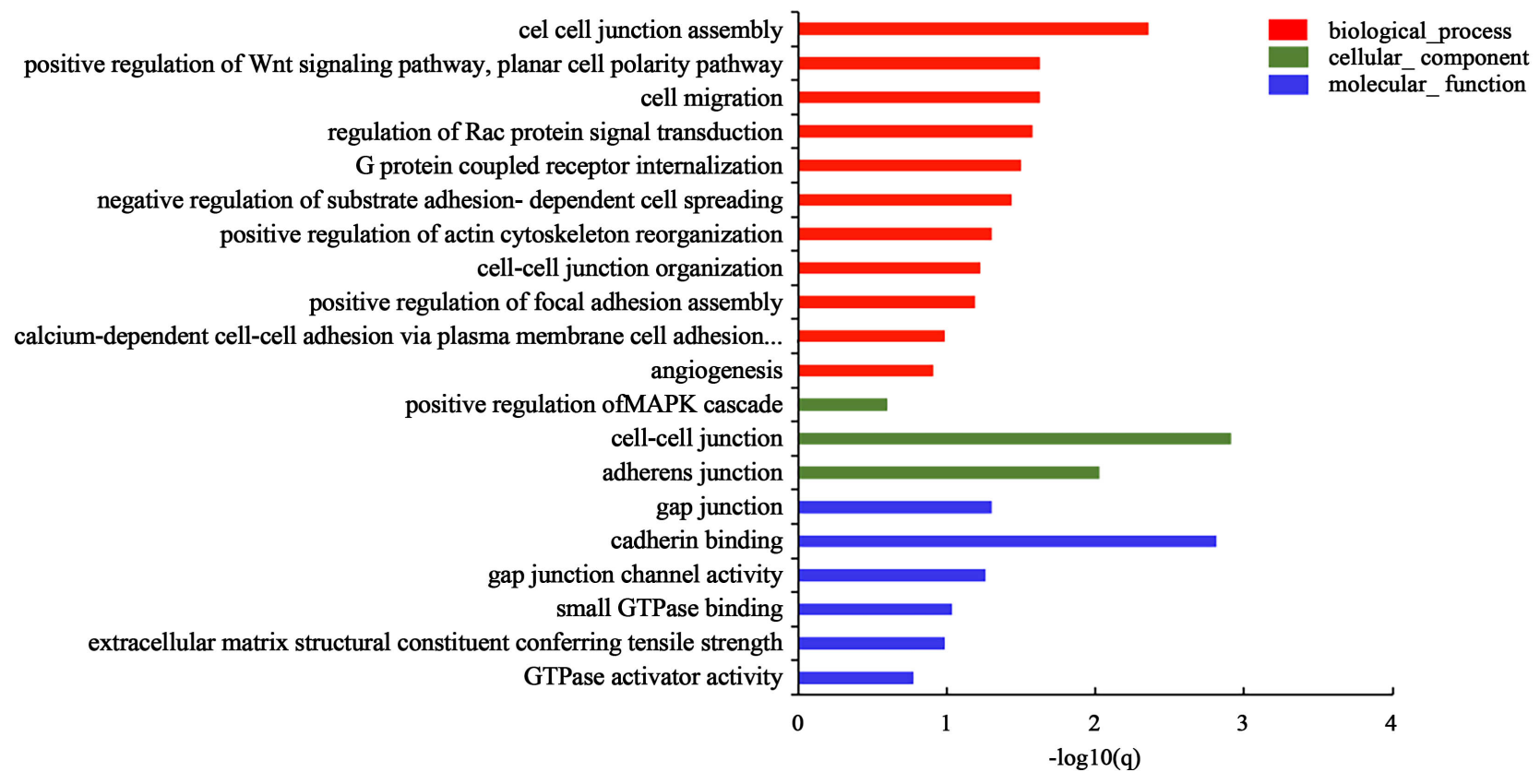

Figure 1. Substrate stiffness regulated cell-cell junctions and the classical signal transduction pathway MAPK. Gene Ontology (GO) annotation analysis of differentially expressed genes. The top 20 GO items were selected according to the order of significance. 


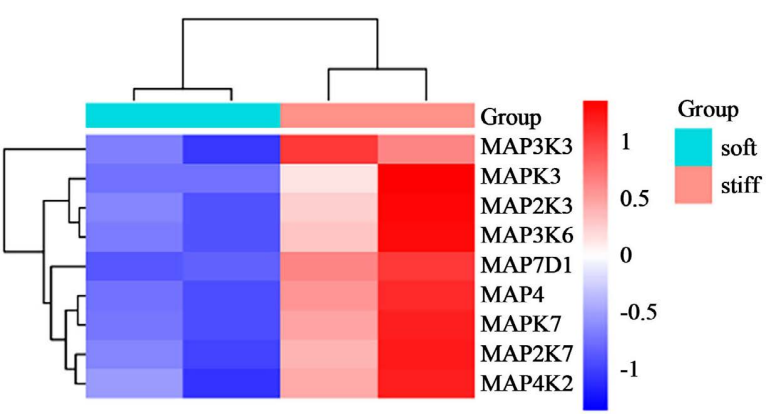

(a)

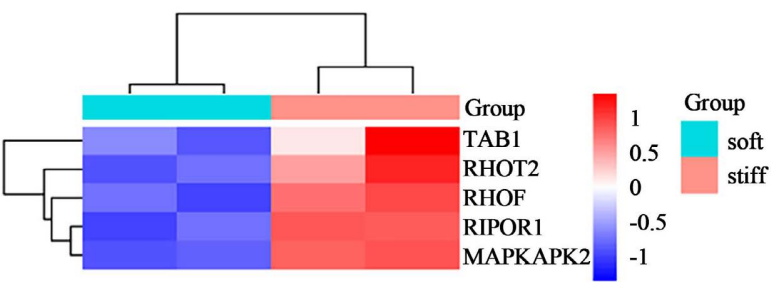

(b)

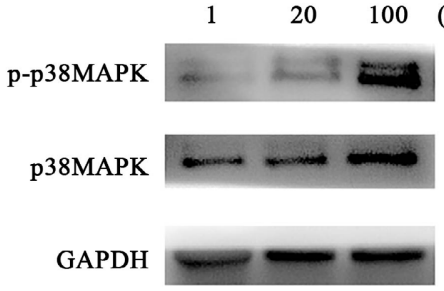

(c)

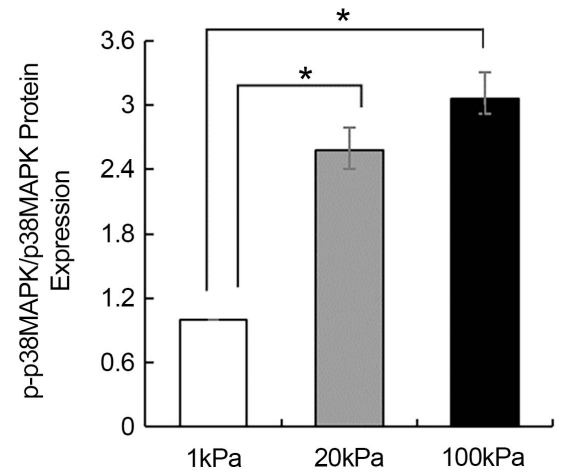

(d)

Figure 2. Substrate stiffness regulated MAPK signaling pathway (a) Transcriptome analysis of MAPK-related molecules by culturing ECs on softer $(1 \mathrm{kPa})$ and stiffer $(100 \mathrm{kPa})$ substrates. (b) Transcriptome analysis of molecules related to MAPK activation by culturing ECs on softer $(1 \mathrm{kPa})$ and stiffer $(100 \mathrm{kPa})$ substrates. (c) The protein expression level of p-p38MAPK and p38MAPK in ECs. (d) The ratio of protein expression p-p38MAPK/p38MAPK $(n=3),{ }^{*} \mathrm{p}<0.05$.

cultured on softer $(1 \mathrm{kPa})$ and stiffer $(100 \mathrm{kPa})$ substrates revealed that the expression of MAPK signaling pathway-related molecules MAP2K3, MAP2K7, MAP3K3, MAP3K6, MAPK3, MAPK7, as well as RHO family interacting cell polarization regulator 1 (RIPOR1), ras homolog family member (RHOF), ras 
homolog family member related to GTPase activity T2 (RHOT2), TGF- $\beta$ activated kinase 1 (MAP3K7) binding protein 1 (TAB1) and MAPK activated protein kinase 2 (MAPKAPK2), which are molecules related to MAPK activation, were also upregulated with increased stiffness (Figure 2(a), Figure 2(b)). The ratio of protein expression $\mathrm{p}-\mathrm{p} 38 \mathrm{MAPK} / \mathrm{p} 38 \mathrm{MAPK}$ was found to increase with stiffness by culturing ECs in vitro at three stiffnesses of $1 \mathrm{kPa}, 20 \mathrm{kPa}$ and 100 $\mathrm{kPa}$ (Figure 2(c), Figure 2(d)).

\subsection{Expression of EC Junctions Related-Genes Decreased on Higher Substrate Stiffness}

Cell junctions assume key roles in pathological processes, which confer resilience and stiffness to tissues, mediate signal transmission, control the transport of ions and molecules, and act as channels to mediate the transport of ions and molecules. Tight junctions (TJs), anchoring junctions, and gap junctions play different roles in pathological processes. Zona occludens1 (ZO-1), encoded by the tight junction protein 1 (TJP1) gene, is a regulator of endothelial cell permeability [20]. TJP2, linked to the actin cytoskeleton is an important component of tight junction proteins, which is also associated with signaling pathways [21]. Junctional adhesion molecules (JAMs) are involved in different cellular functions, such as regulating the formation of cell junctions, cell migration or mitotic spindle orientation. Thus, all the above proteins play an important role in endothelial barrier formation and angiogenesis [22]. Transcriptome analysis of ECs cultured on softer $(1 \mathrm{kPa})$ and stiffer $(100 \mathrm{kPa})$ substrates revealed that the expression of cell junction-related molecules TJP1, TJP2, JAM3 and JCAD were reduced on stiffer substrates (Figure 3(a)). Junctional Cadherin 5 Associated (JCAD) is an endothelial cell ligand protein that regulates pathological angiogenesis, EC proliferation and migration, and the CAD/MI risk gene JCAD identified by Genome-wide association study (GWAS) promotes endothelial dysfunction and atherosclerosis in mice [23]. We found that the gene expression level of JCAD showed a negative correlation to stiffness (Figure 3(b)).

\subsection{The Integrity of Cell-Cell Junctions Was Impaired on High Substrate Stiffness}

VE-cadherin is an important component of EC adherent junctions and has a key role in maintaining vascular integrity by controlling vascular permeability and inhibiting unrestricted vascular growth. It was found that its changes may involve endothelial cytoskeleton remodeling and regulation of gene transcription, etc. Therefore, we performed immunofluorescence staining for VE-cadherin (Figure 4). Immunostaining results showed the punctuation and discontinuity of cell-cell junction (as indicated by red arrows) tended to be increased (Figure 4(a)). The total fluorescence intensity of VE-cadherin in ECs differed significantly between softer and stiffer substrates. The quantitative analysis of fluorescence intensity demonstrated that the expression level of VE-cadherin decreased significantly with increased substrate stiffness (Figure $4(\mathrm{~b})$ ). The above results 
suggested that high substrate stiffness impaired endothelial cell-cell junctions and the integrity of endothelial cell.

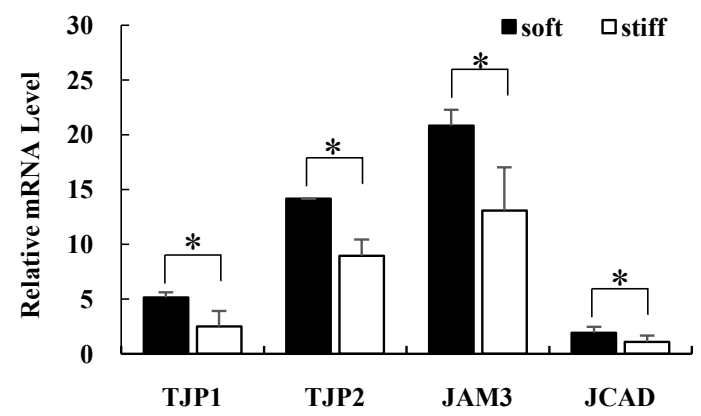

(a)

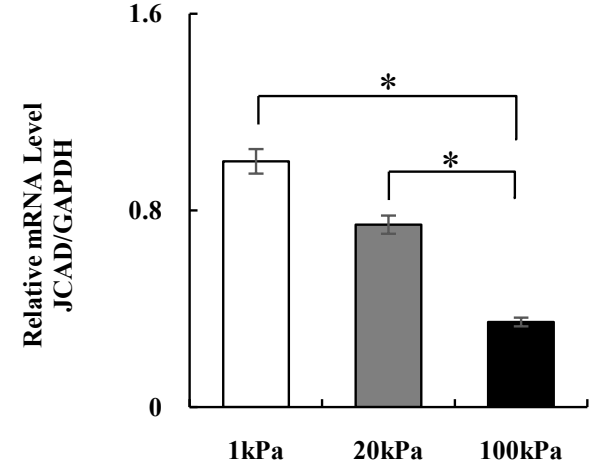

(b)

Figure 3. Expression of endothelial cell junctions related-genes decreased on higher substrate stiffness. (a) Transcriptome analysis of cell junction-related molecules by culturing ECs on softer $(1 \mathrm{kPa})$ and stiffer $(100 \mathrm{kPa})$ substrates, including TJP1, TJP2, JAM3 and JCAD $(n=2)$. (b) The mRNA expression levels of JCAD in HUVECs cultured on $1 \mathrm{kPa}, 20 \mathrm{kPa}$ and $100 \mathrm{kPa}$ substrate was analyzed by qRT-PCR. GAPDH was used as the internal control $(n=3),{ }^{*} \mathrm{p}<0.05$.

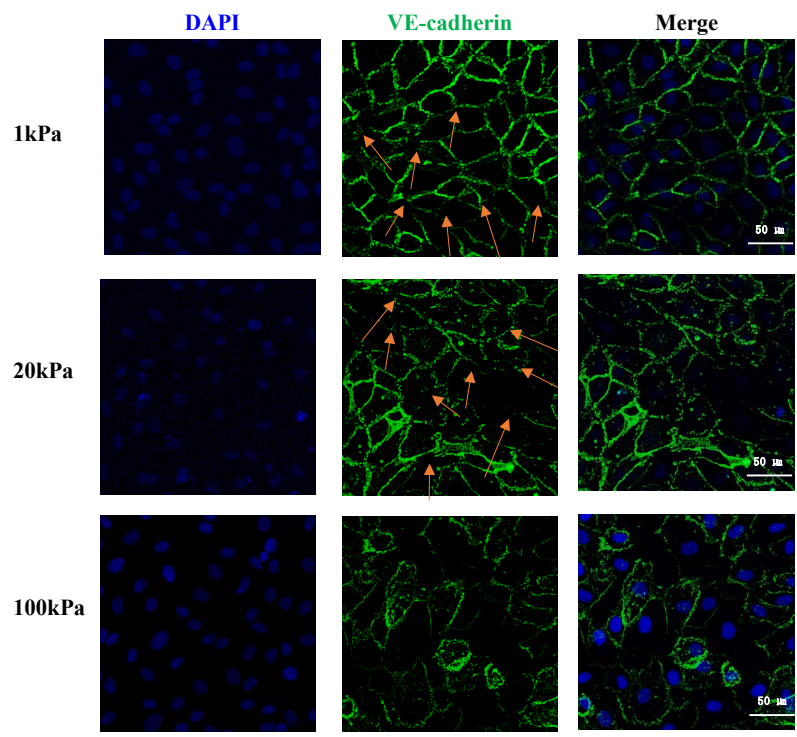

(a) 


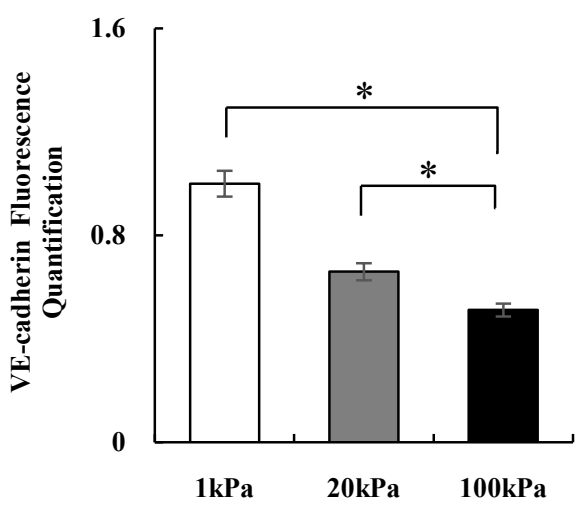

(b)

Figure 4. The integrity of cell-cell junctions was impaired on higher substrate stiffness. (a) Immunofluorescence staining of VE-cadherin (green) and DAPI (blue) in HUVECs cultured on $1 \mathrm{kPa}, 20 \mathrm{kPa}$ and $100 \mathrm{kPa}$ substrate.

(b) The fluorescence quantification of VE-cadherin $(n=3)$, ${ }^{*} p<0.05$.

\section{Discussion}

Blood vessels exhibit a remarkable ability to adapt throughout life that depends upon genetic programming and well-orchestrated biochemical processes. Aging, atherosclerosis, and fibrosis, structural adjustment of the microenvironment in pathological location are often accompanied by changes in tissue stiffness which may cause activation of signaling pathways and cellular dysfunction. Differences in composition and structure result in different extracellular matrix stiffness. When the extracellular matrix stiffness increased, the cells respond to elevated mechanical stress, which may cause altered expression of some genes, damaged endothelial barrier function and blood vessel malfunction.

Both mechanosensitive transcription factors and mechanosensitive ion channels are important factors in vascular sclerosis associated diseases. Mechanosensitive factors include KLF2, KLF4, NRF2, YAP/TAZ/TEAD, NF- $\kappa$ B, and AP-1, which are involved in vascular homeostasis and regulate the expression of various anti-inflammatory, antioxidant, and antithrombotic genes in ECs, as well as metabolic and inflammatory responses related to atherosclerosis [24]. Mechanosensitive ion channels include Transient receptor potential vanilloid-type 4 (TRPV4) and Piezo1, which may activate innate immune cells, increase extracellular matrix deposition and matrix stiffness through mechanical transduction of TRPV4 in fibrotic diseases [25]. Piezo1 is present in ECs and vascular smooth muscle cells, where it is activated by shear stress and cell membrane stretch in response to elevated blood pressure [26]. However, the mechanism of mechanotransduction in vascular sclerosis and homeostasis remains to be investigated.

In this study, we prepared a model of vascular sclerosis using biocompatible polyacrylamide gels. Using this in vitro stiffness model, we found that the expression of TJ-related molecules TJP1, TJP2, JAM3 and JCAD demonstrated a negative correlation with stiffness, the higher the stiffness, the lower the expression of the TJ proteins. This is in agreement with previous studies, that the ex- 
pression, distribution, and structure of TJs are altered in response to pathological conditions and external stimuli. It has been reported that the expression of JCAD is regulated by blood flow, with increased expression in turbulent regions, and increased expression of JCAD promotes atherosclerosis [27]. We found that the increased stiffness can also decrease the expression of JCAD. VE-cadherin is important for the maintenance and regulation of endothelial cell-cell junctions, vascular permeability and leukocyte extravasation. Deficiency of VE-cadherin in humans leads to impaired endothelial cell-cell junctions. Studies also suggested that VE-cadherin adhesion may play an upstream role in the maintenance of endothelial barrier integrity [28]. We found that VE-cadherin showed punctuation and discontinuity in cell-cell junctions, suggesting the integrity of endothelial cell-cell junctions being impaired by high substrate stiffness. MAPK family plays key roles in cardiovascular development and vascular function, and MAPK activation in vascular tissues is age-dependent [29] [30] [31] [32]. We also found that the expression of MAPK signaling pathway-related kinases, and MAPK activation-related molecules were upregulated with increased stiffness, suggesting that MAPK signaling pathway was involved in stiffness related mechanotransduction.

In summary, we found that substrate stiffness played an important role in regulating EC junction integrity. The study of substrate stiffness on EC junction integrity and signaling pathways facilitate further investigation of age-related vascular homeostasis imbalance and other endothelial dysfunctions.

\section{Conflicts of Interest}

The authors declare no conflicts of interest regarding the publication of this paper.

\section{References}

[1] Augustin, H.G. and Koh, G.Y. (2017) Organotypic Vasculature: From Descriptive Heterogeneity to Functional Pathophysiology. Science, 357, Article No. eaal2379. https://doi.org/10.1126/science.aal2379

[2] Greten, F.R. and Grivennikov, S.I. (2019) Inflammation and Cancer: Triggers, Mechanisms, and Consequences. Immunity, 51, 27-41. https://doi.org/10.1016/j.immuni.2019.06.025

[3] Ungvari, Z., Tarantini, S., Donato, A.J., Galvan, V. and Csiszar, A. (2018) Mechanisms of Vascular Aging. Circulation Research, 123, 849-867. https://doi.org/10.1161/CIRCRESAHA.118.311378

[4] Gimbrone, M.J. and García-Cardeña, G. (2016) Endothelial Cell Dysfunction and the Pathobiology of Atherosclerosis. Circulation Research, 118, 620-636. https://doi.org/10.1161/CIRCRESAHA.115.306301

[5] Bachschmid, M.M., Schildknecht, S., Matsui, R., Zee, R., Haeussler, D., Cohen, R.A., et al. (2013) Vascular Aging: Chronic Oxidative Stress and Impairment of Redox Signaling-Consequences for Vascular Homeostasis and Disease. Annals of Medicine, 45, 17-36. https://doi.org/10.3109/07853890.2011.645498

[6] Rajendran, P., Rengarajan, T., Thangavel, J., Nishigaki, Y., Sakthisekaran, D., Sethi, 
G., et al. (2013) The Vascular Endothelium and Human Diseases. International Journal of Biological Sciences, 9, 1057-1069. https://doi.org/10.7150/ijbs.7502

[7] Reina-Torres, E., De Ieso, M.L., Pasquale, L.R., Madekurozwa, M., van Batenburg-Sherwood, J., et al. (2021) The Vital Role for Nitric Oxide in Intraocular Pressure Homeostasis. Progress in Retinal and Eye Research, 83, Article ID: 100922. https://doi.org/10.1016/j.preteyeres.2020.100922

[8] Loader, J., Montero, D., Lorenzen, C., Watts, R., Méziat, C., Reboul, C., et al. (2015) Acute Hyperglycemia Impairs Vascular Function in Healthy and Cardiometabolic Diseased Subjects: Systematic Review and Meta-Analysis. Arteriosclerosis, Thrombosis, and Vascular Biology, 35, 2060-1072. https://doi.org/10.1161/ATVBAHA.115.305530

[9] Oikonomou, E., Siasos, G., Tsigkou, V., Bletsa, E., Panoilia, M.E., Oikonomou, I.N., et al. (2020) Coronary Artery Disease and Endothelial Dysfunction: Novel Diagnostic and Therapeutic Approaches. Current Medicinal Chemistry, 27, 1052-1080. https://doi.org/10.2174/0929867326666190830103219

[10] Semenza, G.L. (2005) New Insights into nNOS Regulation of Vascular Homeostasis. Journal of Clinical Investigation, 115, 2976-2978. https://doi.org/10.1172/JCI26792

[11] Schinzari, F., Tesauro, M. and Cardillo, C. (2017) Vascular Hyperpolarization in Human Physiology and Cardiovascular Risk Conditions and Disease. Acta Physiologica, 219, 124-137. https://doi.org/10.1111/apha.12630

[12] Yurdagul, A.J., Finney, A.C., Woolard, M.D. and Orr, A.W. (2016) The Arterial Microenvironment: The Where and Why of Atherosclerosis. Biochemical Journal, 473, 1281-1295. https://doi.org/10.1042/BJ20150844

[13] Costantino, S., Paneni, F. and Cosentino, F. (2016) Ageing, Metabolism and Cardiovascular Disease. The Journal of Physiology, 594, 2061-2073. https://doi.org/10.1113/JP270538

[14] Jia, G., Aroor, A.R., Jia, C. and Sowers, J.R. (2019) Endothelial Cell Senescence in Aging-Related Vascular Dysfunction. Biochimica et Biophysica Acta (BBA): Molecular Basis of Disease, 1865, 1802-1809. https://doi.org/10.1016/j.bbadis.2018.08.008

[15] Akhtar, R., Graham, H.K., Derby, B., Sherratt, M.J., Trafford, A.W., Chadwick, R.S., et al. (2016) Frequency-Modulated Atomic Force Microscopy Localises Viscoelastic Remodelling in the Ageing Sheep Aorta. Journal of the Mechanical Behavior of Biomedical Materials, 64, 10-17. https://doi.org/10.1016/j.jmbbm.2016.07.018

[16] Vestweber, D. (2008) VE-Cadherin: The Major Endothelial Adhesion Molecule Controlling Cellular Junctions and Blood Vessel Formation. Arteriosclerosis, Thrombosis, and Vascular Biology, 28, 223-232. https://doi.org/10.1161/ATVBAHA.107.158014

[17] Fischer, R.S., Myers, K.A., Gardel, M.L. and Waterman, C.M. (2012) Stiffness-Controlled Three-Dimensional Extracellular Matrices for High-Resolution Imaging of Cell Behavior. Nature Protocols, 7, 2056-2066.

https://doi.org/10.1038/nprot.2012.127

[18] Chen, W., Tian, B., Liang, J., Yu, S., Zhou, Y. and Li, S. (2019) Matrix Stiffness Regulates the Interactions between Endothelial Cells and Monocytes. Biomaterials, 221, Article ID: 119362. https://doi.org/10.1016/j.biomaterials.2019.119362

[19] Tian, B., Ding, X., Song, Y., Chen, W., Liang, J., Yang, L., et al. (2019) Matrix Stiffness Regulates SMC Functions via TGF- $\beta$ Signaling Pathway. Biomaterials, 221, Article ID: 119407. https://doi.org/10.1016/j.biomaterials.2019.119407

[20] Dai, W., Nadadur, R.D., Brennan, J.A., Smith, H.L., Shen, K.M., Gadek, M., et al. 
(2020) ZO-1 Regulates Intercalated Disc Composition and Atrioventricular Node Conduction. Circulation Research, 127, e28-e43. https://doi.org/10.1161/CIRCRESAHA.119.316415

[21] Xu, J., Kausalya, P.J., Van Hul, N., Caldez, M.J., Xu, S., Ong, A.G.M., Woo, W.L., et al. (2021) Protective Functions of ZO-2/Tjp2 Expressed in Hepatocytes and Cholangiocytes against Liver Injury and Cholestasis. Gastroenterology, 160, 2103-2118. https://doi.org/10.1053/j.gastro.2021.01.027

[22] Ebnet, K. (2017) Junctional Adhesion Molecules (JAMs): Cell Adhesion Receptors with Pleiotropic Functions in Cell Physiology and Development. Physiological Reviews, 97, 1529-1554. https://doi.org/10.1152/physrev.00004.2017

[23] Xu, S., Xu, Y., Liu, P., Zhang, S., Liu, H., Slavin, S., Kumar, S., et al. (2019) The Novel Coronary Artery Disease Risk Gene JCAD/KIAA1462 Promotes Endothelial Dysfunction and Atherosclerosis. European Heart Journal, 40, 2398-2408. https://doi.org/10.1093/eurheartj/ehz303

[24] Niu, N., Xu, S., Xu, Y., Little, P.J. and Jin, Z.G. (2019) Targeting Mechanosensitive Transcription Factors in Atherosclerosis. Trends in Pharmacological Sciences, 40, 253-266. https://doi.org/10.1016/j.tips.2019.02.004

[25] Michalick, L. and Kuebler, W.M. (2020) TRPV4-A Missing Link between Mechanosensation and Immunity. Frontiers in Immunology, 11, Article No. 413. https://doi.org/10.3389/fimmu.2020.00413

[26] Albarrán-Juárez, J., Iring, A., Wang, S., Joseph, S., Grimm, M., Strilic, B., et al. (2018) Piezo1 and $G_{q} / G_{11}$ Promote Endothelial Inflammation Depending on Flow Pattern and Integrin Activation. Journal of Experimental Medicine, 215, 2655-2672. https://doi.org/10.1084/jem.20180483

[27] Douglas, G., Mehta, V., Al Haj Zen, A., Akoumianakis, I., Goel, A., Rashbrook, V.S., et al. (2020) A Key Role for the Novel Coronary Artery Disease Gene JCAD in Atherosclerosis via Shear Stress Mechanotransduction. Cardiovascular Research, 116, 1863-1874. https://doi.org/10.1093/cvr/cvz263

[28] Giampietro, C., Taddei, A., Corada, M., Sarra-Ferraris, G.M., Alcalay, M., Cavallaro, U., et al. (2012) Overlapping and Divergent Signaling Pathways of N-Cadherin and VE-Cadherin in Endothelial Cells. Blood, 119, 2159-2170.

https://doi.org/10.1182/blood-2011-09-381012

[29] Yang, G., Li, T., Xu, J., Peng, X. and Liu, L. (2013) Mitogen-Activated Protein Kinases Regulate Vascular Reactivity after Hemorrhagic Shock through Myosin Light Chain Phosphorylation Pathway. Journal of Trauma and Acute Care Surgery, 74, 1033-1043. https://doi.org/10.1097/TA.0b013e31828586a2

[30] Pearson, G., Robinson, F., Beers Gibson, T., Xu, B.E., Karandikar, M., Berman, K., et al. (2001) Mitogen-Activated Protein (MAP) Kinase Pathways: Regulation and Physiological Functions. Endocrine Reviews, 22, 153-183.

https://doi.org/10.1210/er.22.2.153

[31] Roux, P.P. and Blenis, J. (2004) ERK and p38 MAPK-Activated Protein Kinases: A Family of Protein Kinases with Diverse Biological Functions. Microbiology and Molecular Biology Reviews, 68, 320-344.

https://doi.org/10.1128/MMBR.68.2.320-344.2004

[32] Rice, K.M., Kinnard, R.S., Harris, R., Wright, G.L. and Blough, E.R. (2005) Effects of Aging on Pressure-Induced MAPK Activation in the Rat Aorta. Pflügers Archiv, 450, 192-199. https://doi.org/10.1007/s00424-005-1383-9 